\title{
Smart Radio Control System (for Flight Test Centers)
}

\author{
Jesus Alvarez \\ Airbus D\&S - Flight Test - Spain
}

\section{Abstract}

Among the rich infrastructure of a Telemetry/Ground Station Center dwells the subset dedicated to radio communications.

Radios are mainly used to communicate with the aircraft under test in order to give guidance and feedback from ground specialists. Sometimes, however, radios themselves become the subject of the test, requiring a full set of them with all their features and capabilities (Military Modes, HF ALE, SELCAL, etc).

Remote control (and audio routing) of these radios is a critical as infrastructures scale over tens of radios, distributed amid different test centers separated by hundreds of kilometers.

Addition of a remote touch user interface, MIL COMSEC and TRANSEC modes, automatic audio routing, together with a maintenance free requirement, makes the whole issue far more difficult to manage.

Airbus Military has developed a Smart Radio Control System allowing to profit from those advantages and more benefits:

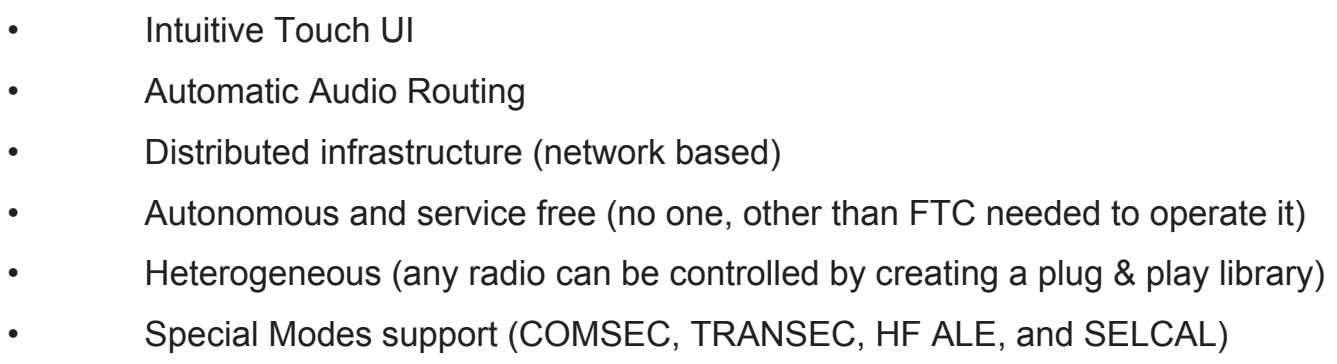

Future additions will include, amongst others, VolP integration and tablet use.

Key words: radio network smart control touch interface distributed system scalable heterogeneous.

\section{Introduction}

A Flight Test Ground Station (GS onwards) or Telemetry Station provides support to Test Flights by receiving real time telemetry from the Aircraft and forwarding engineers feedback back to the Aircraft Crew.

In order to communicate with the Aircraft, the GS uses radio communication equipment, radios, in short.

Radio Communications in aeronautical world refers to those electromagnetic transmissions carrying voice or data in an Air-to-Air or Ground-to-Air scenario.

GS needs to put in place a distributed network of radios all over the flight test zone.
1. Radio used a s a communication mean to provide guidance and support to onboard crew

2. Radio used as a Test Means in a dedicated Communication Flight Test

Former is usually requested for special modes testing as TRANSEC, COMSEC and HF ALE or for range tests.

The creation of such a distributed and remotely controlled radio network is real challenge in terms of control and management.

\subsection{Main challenges \\ Technical}

There are two different usage scenarios: 
- Wide range of devices with different control interfaces/protocols

- Remote locations and sharing of resources

- Concurrent access

- Remote control

- Remote Audio

- Radio Selection

- Special Modes

Usage

- One-person operation

- Click \& go. Service free and setup free

- Intuitive and quick touch interface

\subsection{Equipment Architecture}

In order to build the network of radios, a group of requirements must be defined.

Once the requirements are clear, and based upon them, the equipment selection phase can begin.

1. Any radio can be selected by the user

a. A user can select and use whichever radio he wants to use.

b. This implies control hangover and audio switching mechanism

c. Audio Analog Matrix as the main audio switching method.

2. Remote operation

a. Radios and sites can be hundreds kilometers apart

b. Therefore radios and all other equipment shall have remote control. IP preferred.

3. Robustness $=>$ Limit complexity

a. Avoid complexity.

b. In this particular case and regarding audio routing, only Audio Matrix will be controlled to change audio ties. VolP ties will always be fixed

4. Radio Control System has to be flexible, inexpensive, future proof

a. Control architecture based on PCs

b. Own HW \& SW development

\subsection{Key Elements}

- FTC

Flight Test Conductor. He is the person that communicates with the Aircraft via radio to provide assistance. Therefore he is the main user of the Radio Control System

- Radio

The actual radio

- VolP Gateway

Equipment used to convert analog voice to $I P$, route it through the IP network and convert it back to analog at the other peer.

- Smart Radio Control System

Automatic SW based control system that controls and manages every single aspect of the radio network allowing a user to use any of the radios within it.

- Audio Matrix

Main audio routing / switching equipment

\subsection{Basic Interconnection}

Based on this equipment, a generic local interconnection schema could be as follows:

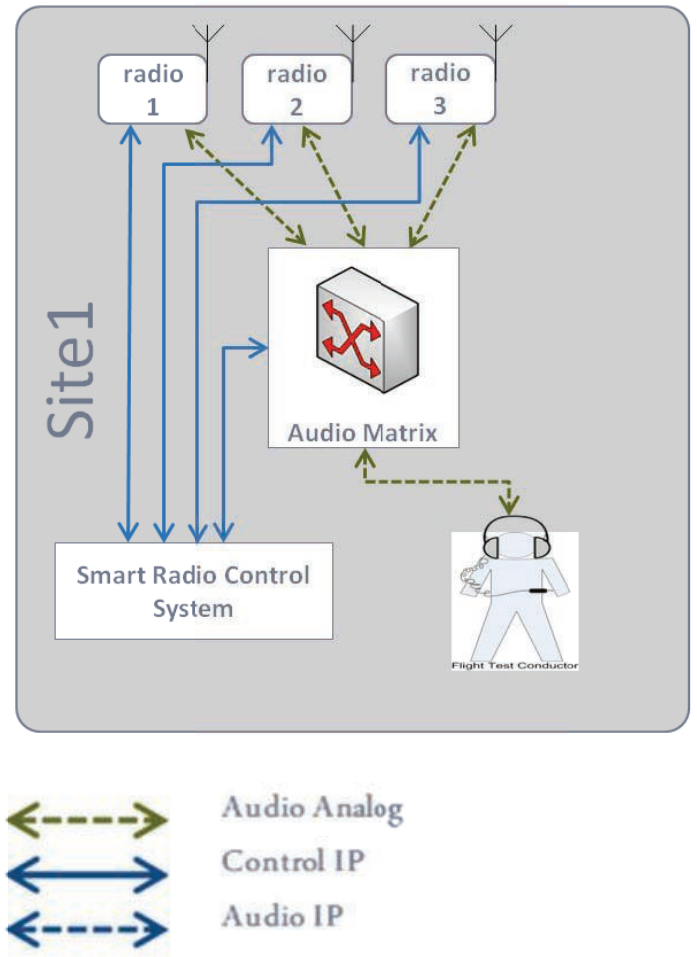

The above interconnection is the simplest one as there is no remote site control and audio link and number of radios to control is still low. 
In the following picture we have added a remote

VolP equipment is added.

radio to see how complexity grows at the time

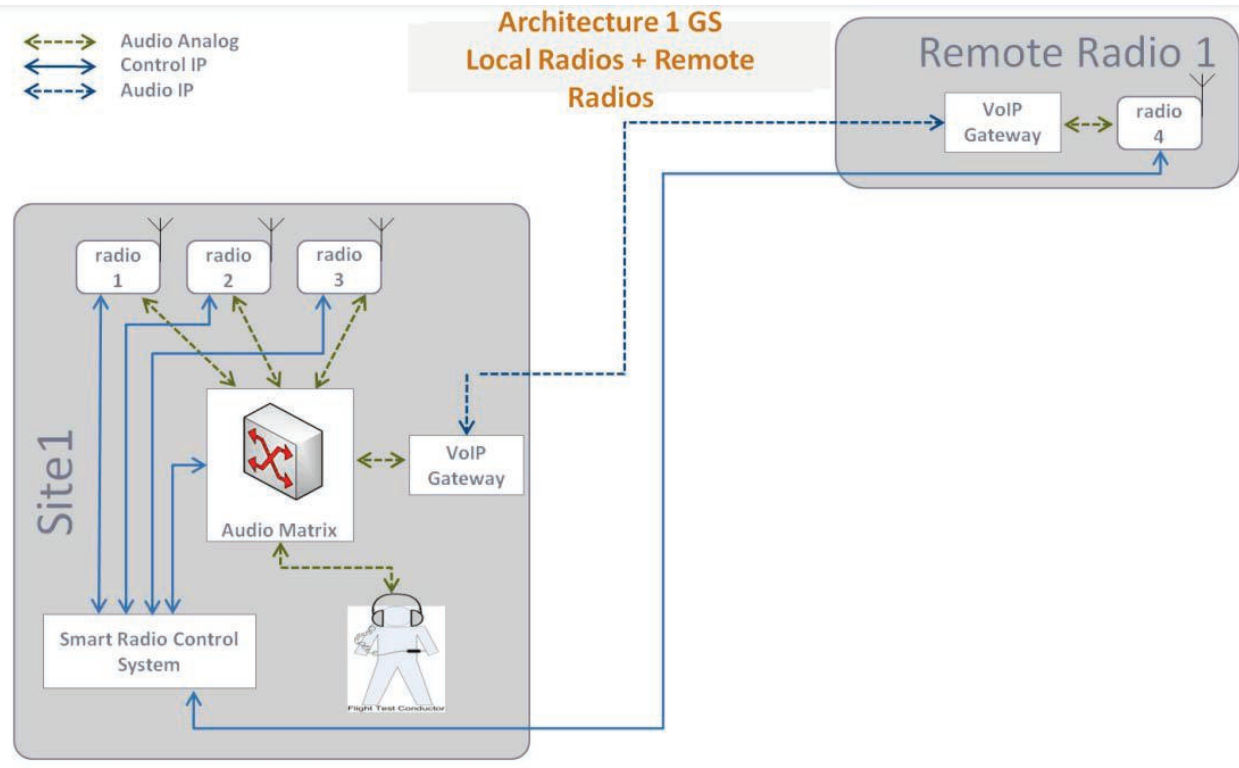

Thanks to the VolP fixed link, the radio is

Even if the VolP equipment has been added to interface and transport voice to the remote radio, the audio switching still remains at the local Audio Matrix. actually considered by the system as it were a local radio.

One step further is shown in the next picture.

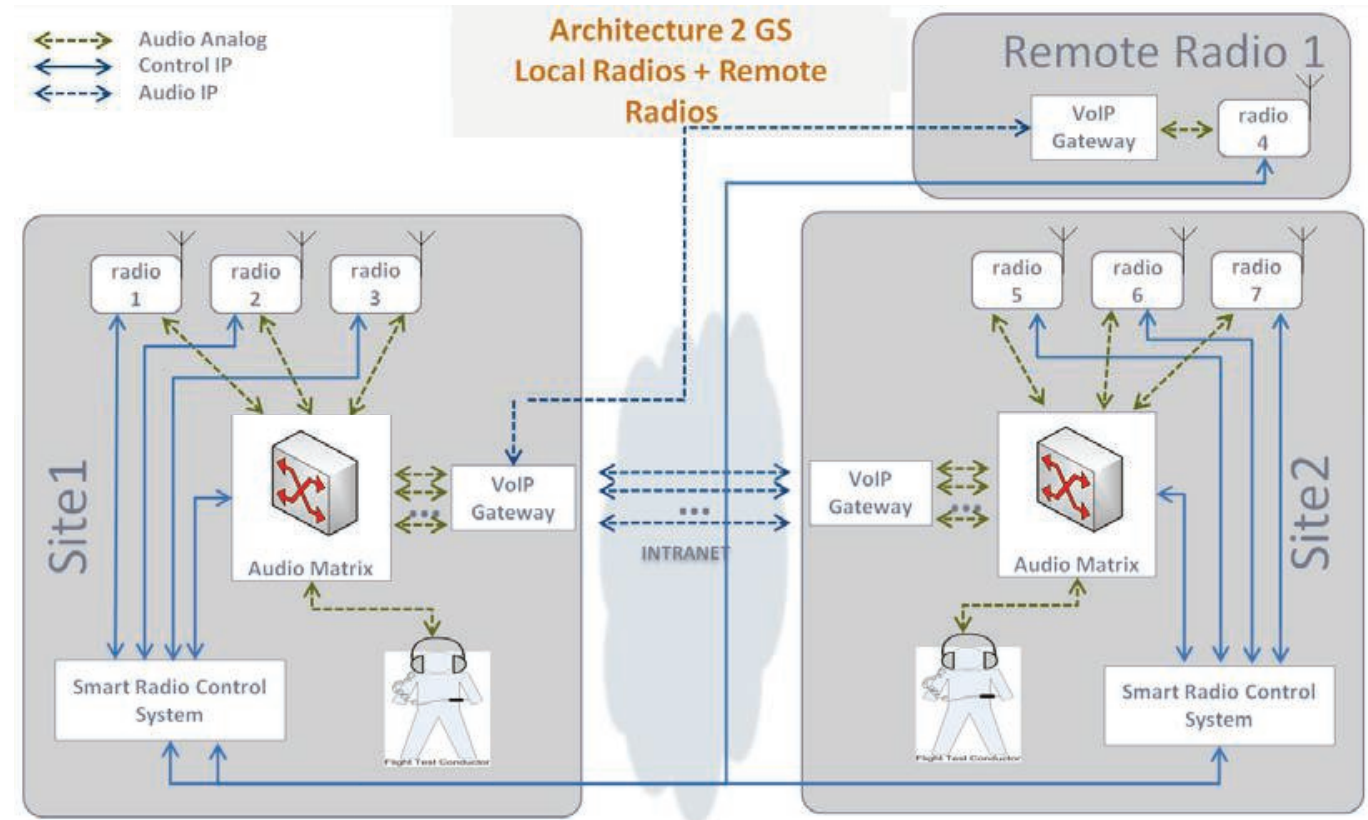

As the reader can notice, complexity has grown again.

This interconnection schema represents two GS plus one remote radio network.

Remote radio 1 is still seen as a local radio to Site 1. Therefore if Site2 needs to use it, two audio ties will have to be performed at both Audio Matrix Site 1 and Site 2.

Same situation if Site 1 wants to use a radio from Site 2, two audio ties are needed. 


\section{Smart Radio Control System}

It can be seen as an integrated software and hardware system that allows the operation and management of a network of radios in a transparent manner.

\subsection{Features}

\section{Unattended}

1. It does not need daily administration

This is quite important as it allows to free operators from giving radio support to users

2. Audio routing is transparent and automatic

Audio routing is calculated and commanded automatically by the system. There is no operator intervention or manual action required.

\section{Integrated}

3. Every radio on the network can be included

4. Every radio is operable from any location

Once the VolP lines are installed, every radio can be used from any location and it is as easy a one-click radio selection in the HMI.

\section{Heterogeneous}

5. Any radio with remote control can be added

The SW system works with radio control libraries (DLL).

Every radio can be controlled as far as it provides a remote control interface.

In terms of interfaces they can go from a simple PTT relay (for a non controllable radio) to Serial, GPIO or IP interface. In the case of Serial and GPIO, an IP converter is used.

6. Radios from different manufacturers: Rohde, PAE, Rockwell Collins, SunAir

Intuitive

7. Graphical interface easy and simple to use... eye-candy

\section{Customizable (per user)}

8. Radios lay-out

User can change the radio lay out in the HMI to his convenience.

9. Quick frequency presets

User can define quick access presets in the HMI

\subsection{Design Phase - Requirements and Constraints}

- Distributed. Avoid radio block. Manage multi access.

In order to fulfill this requirement, the software model should be client-server.

This way there will be few servers that have unique access to a given radio and as many as needed clients that access radio features through requests to servers

- Client interface intuitive, fast and nonintrusive

Radio Client $\mathrm{HMI}$ will have to be located on the FTC desk.

Operation of radios shall be as fast and non intrusive as possible. FTC has enough displays and work to do during a Flight Test.

To comply with these requirements, a small touch screen seemed to be a good option. Therefore the SW UI has to be finger friendly

- $\quad$ Physical PTT

FTCs are used to physical PTT mechanism so the system should allow using them.

A physical PTT is no more than a contact closure that has to be detected by the Radio Server in order to command PTT to the selected radio.

This requirement imposes the use of PCs with parallel port for the server.

- $\quad$ Robust. Limit complexity

In order to limit complex connection management, the use of UDP/IP protocol is adopted in LAN. Multicast type if possible.

- WAN friendly

In order to work correctly in a WAN environment were packet loss can occur; the servers' interconnections shall be done using TCP/IP protocol

\section{- $\quad$ Radio Control Interface agnostic}

By using IP converters and SW layers, any radio can be controlled by the system. 


\subsection{Key Elements}

Among the key elements of the system:

- $\quad$ FTC

Flight test conductor

- Radio

The actual radio

- $\quad$ PTT Switches

Physical switches used by the user to activate PTT on the selected radio

- PC Server

PC Windows with the Smart Radio Control Server SW installed. It must have a parallel interface

- $\quad$ PC Client with Touch screen

PC Windows with the Smart Radio Control Client SW installed and a touch screen.

\subsection{Basic Operation}

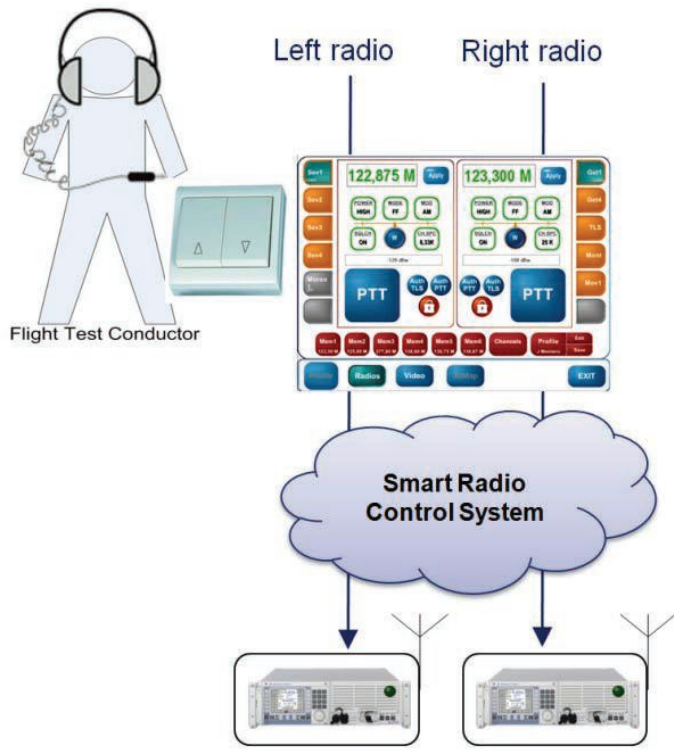

In the previous picture the reader can see the basic operation of the system.

Two radios can be operated at the same time by the user, one on the left and one on the right part of the screen.

As mentioned, the screen is split in two parts, left and right side. The selected radio on the left is associated with the physical PTT switch on the left and the selected radio on the right is associated with the physical PTT switch on the right.

PTT can be activated by both physical PTT and touch screen PTT button.

\subsection{Software Design}

\subsubsection{Client - Server Mode}

In order to fulfill the requirements of multiple users and locations, a client - server model was chosen.

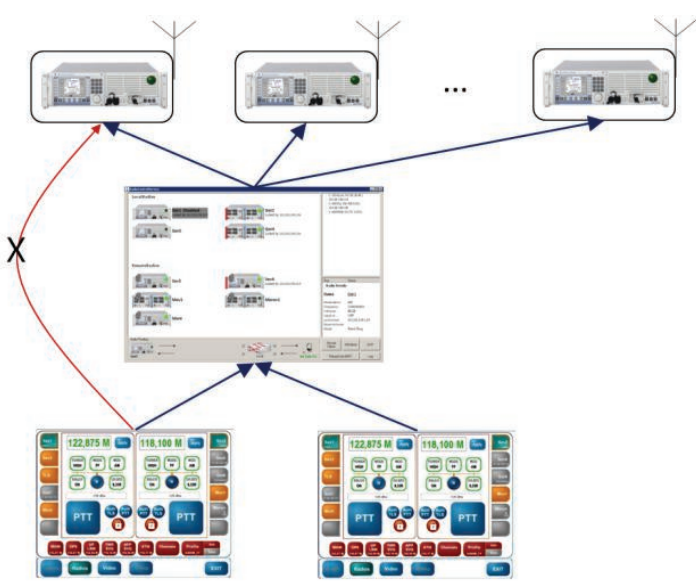

Only the assigned server talks directly to the radio. Clients just send commands and request to servers that decide and orchestrate their execution on the radios.

\subsubsection{Audio Routing}

Audio routing is done by commanding the Audio Matrixes to tie several inputs to several outputs in all required locations.

This process is initiated at the moment a radio is selected by the user.

Audio routing status and information can be monitored at any time in the Server HMI.

Following images show the audio switching process for a local and a remote radio.
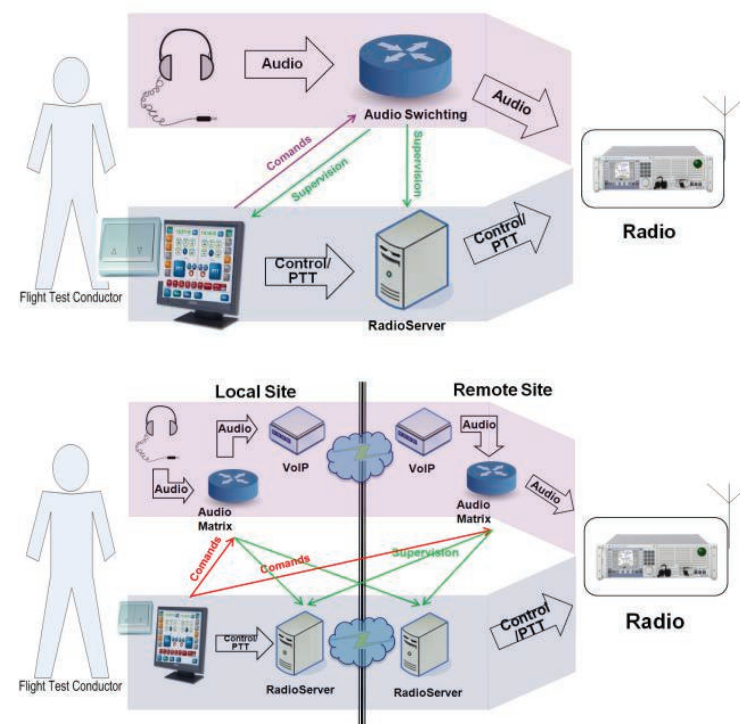


\subsubsection{Software Architecture}

Software was designed to be scalable and easy to maintain.

It is configured in isolated layers separated by common interfaces. This way, a change in a layer does not affect another one.

\section{Key features}

- Common Standard Protocol C-S

A common and isolated protocol was put in place for Client - Server communications.

This protocol is radio agnostic and should need no changes

- Isolation from Device specific communications interface/protocol

- SW Library per device type

A specific software communications library is created for every radio type. This library implements a common interface that will be explained afterwards.

\section{Software components}

Software layers are shown in the following picture.

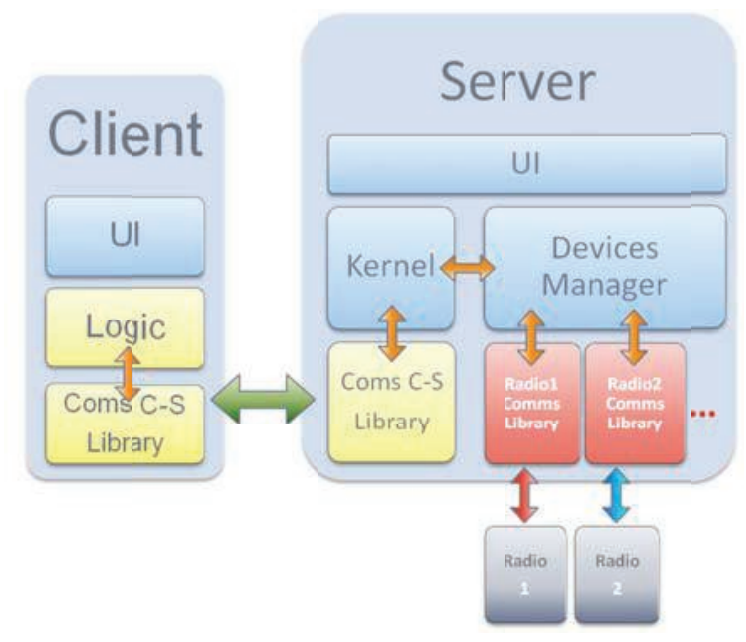

- $\quad$ Client

- Client-Server Communications Library

Common interface and protocol between client and server.

It uses UDP if the equipment is in local LAN and TCP if server is in WAN

- Logic Layer

It captures events from communication layer and draws the $\mathrm{UI}$ interface accordingly. At the same time translate user $\mathrm{UI}$ events into commands to the communication layer.

○ UI Layer

It draws the $\mathrm{HMI}$ and generates $\mathrm{UI}$ events to logic layer.

- Server

- Client-Server Communications Library

- Kernel Layer

It manages clients' commands and decides whether they can be sent to radios.

○ Device Manager

It holds a number of communication objects with every local radio and forward kernel messages to the desired radio.

- Per device communiication library

It inherits from "RadioLink" Generic Class allowing a single common interface to the Kernel layer. Simplicity

It is based on Events to Device Manager and Kernel layer, avoiding polling.

\subsubsection{User Interface}

Some screen captures of the $\mathrm{UI}$ are shown below.

\section{Main Interface}

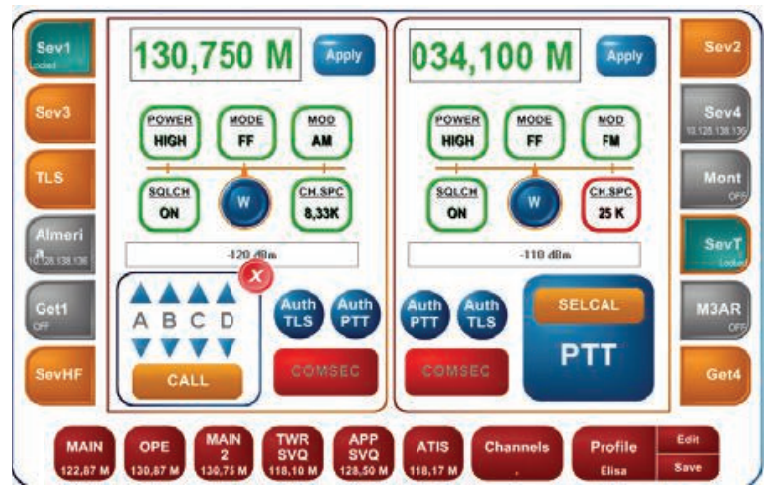

Havequick \& SATURN

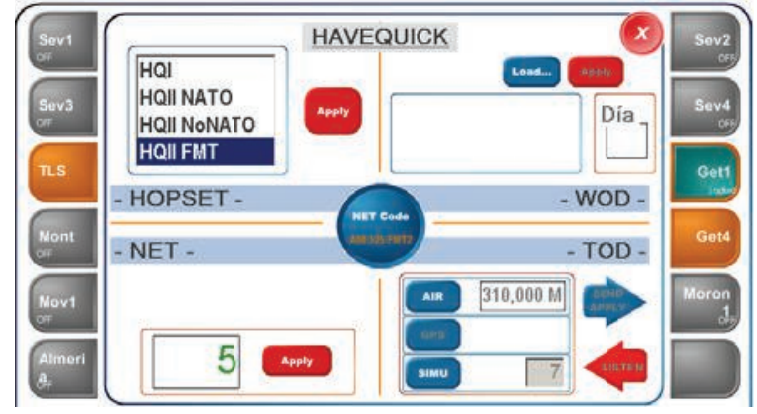

